Z Herz-Thorax- Gefäßchir 2009 • 23:130

DOI 10.1007/s00398-009-0722-0

๑) Springer-Verlag 2009

\author{
H.-R. Zerkowski \\ Riehen, Schweiz
}

\title{
Was und zu welchem Ende lernen wir?
}

Liebe Leserinnen und Leser,

auch Banker haben gelernt - neue Erkenntnisse über Märkte, Transaktionen, Shareholder, Stakeholder, Gewinnmaximierung, Eigenkapitalrendite ... Sie verloren den Partner, den Kunden aus dem Auge. Sie haben das große Ganze und den, der eigentlich im Mittelpunkt steht, vergessen, dafür sich selbst offenbar in ihrer Rolle maximiert. Nun stehen Sie am Pranger. Zur guten, allseits zufrieden stimmenden Berufsausübung gehört also doch immer mehr, als lediglich eine gute Arbeit zu machen. Laufen auch wir nicht Gefahr, in unserem Beruf hinsichtlich verschiedener anderer Gesichtspunkte zu einseitig zu werden?

Wir, die wir doch eigentlich Herzund/oder (je nach Schwerpunkt, Zieloder Wunschvorstellung) Thoraxchirurgen werden wollten - mit der Betonung auf „Chirurg“, also dem Arzt, der mit chirurgischen Methoden behandelt - müssen, oder besser wollen, uns lernend, dazulernend, immer stärker mit Dingen beschäftigen, die so gar nicht in das Berufsbild passen, welches wir uns irgendwann selbst einmal gemacht hatten.

In diesem Heft finden Sie Übersichten zu seltenen Tumoren innerhalb des Fachgebiets, zum Umgang mit gefürchteten Komplikationen, didaktisch aufbereitete Fallberichte ... D'accord. Das ist Weiterbildung.
Aber Strukturfragen, Fiktionen der experimentellen Medizin, theoretisches Grund- und Hintergrundwissen zur Beurteilung von Studien und der Relevanz ihrer Ergebnisse - wozu das?

Wir betreiben ein großartiges Fach, das in Situationen existenzieller Bedrohung durch Erkrankungen chirurgische Lösungen mit nachhaltig guten Ergebnissen bietet.

Die Zeitläufte aber haben sich geändert, der Chirurg ist heute mehr, muss heute mehr sein! Denn obendrein ist auch der Patient (k)ein anderer, sicher aber anders geworden als wir Älteren ihn zu Assistenzzeiten erlebten. Er ist aufgeklärt, mündig im besten Sinne und fordert nachvollziehbare Erklärungen und partnerschaftliches Verhalten ein. Der Chirurg hilft ihm auf seinem Weg durch die und mit der Krankheit - wir erläutern, beraten, raten.

Sie müssen auf Ihrem Weg dem Patienten die Richtung vorgeben, glaubhaft und authentisch sein; nur das zählt.

Dazu müssen wir Strukturen kennen, Wissenschaft beurteilen und transferieren können, Evidenz umsetzen und dies alles unseren Patienten bestmöglich vermitteln.

Ein Chirurg muss operieren können; das darf vorausgesetzt werden. Darüber hinaus muss er aber die Richtung, den Weg zeigen und die Aussicht auf Problemüberwindung oder zumindest -beherr- schung anbieten können - begründet, belastbar, fundiert und kompetent.

Auf dem Weg dorthin soll auch dieses Heft wieder ein kleines Stück weiter helfen.

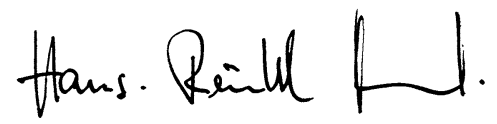

H.-R. Zerkowski

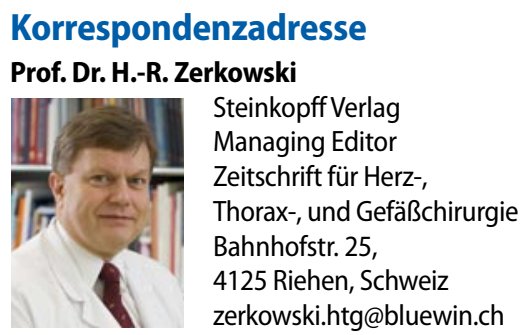

